\title{
Daño neurológico perioperatorio. Identificación de factores de riesgo, prevención y estudios relacionados
}

\author{
Perioperative nerve injury. Risk factor identification, \\ prevention and work-up
}

Marcelo Bernardin M. MD. ${ }^{1}$, Marcela Álvarez E. MD. ${ }^{2}$, Viviana Alam MD. ${ }^{3}$

\begin{abstract}
Background: Regional anesthesia techniques can have adverse effects, like peripheral nerve injuries. This can affect the practitioner on the choice of techniques when offering multimodal analgesia. Case report: We present the case of an arthroscopic rotator cuff repair on a patient with no comorbidities who presented peripheral nerve injury during post-op. Initially thought to have occurred as a consequence of the anesthetic technique, further study of the patient revealed the injuries to have been produced by the arthroscopic surgery. Conclusion: There are multiple factors that influence the possibility of peripheral nerve injury associated with nerve blocks. They can be grouped as related to the patient, the anesthetic technique or the surgical technique, the latter being the most relevant. If a patient manifests signs of peripheral nerve injury high-resolution magnetic resonance of the neural tissue must be performed immediately. Sensory nerve conduction study and electromyography must be performed 4 weeks after by an expert neurologist.
\end{abstract}

\section{RESUMEN}

Introducción: La anestesia regional puede producir como efecto adverso lesiones de nervios periféricos, lo que puede hacer desistir al anestesiólogo de realizar técnicas que mejoran la analgesia multimodal. Caso clínico: Se pre-

\section{Key words:}

Nerve block, peripheral nerve injuries, brachial plexus neuritis, arthroscopy

\section{Palabras clave:}

Bloqueo nervioso,

neuritis del plexo

braquial,

artroscopía,

complicaciones

Staff de Anestesiología Hospital Clínico Mutual. Encargados de Anestesia Regional y Programa de Dolor Agudo Postoperatorio. Profesor Adjunto Anestesia Regional Universidad Chile y Universidad del Desarrollo.

2 Staff de Anestesiología Hospital Clínico Mutual. Encargados de Anestesia Regional y Programa de Dolor Agudo Postoperatorio. Profesor Adjunto Anestesia Regional Universidad Mayor.

3 Staff de Anestesiología Hospital San Juan de Dios. Profesor Adjunto y encargada de Postgrado de Anestesia Universidad Chile.

Fecha de recepción: 30 de octubre de 2019

Fecha de aceptación: 14 de noviembre de 2019

ORCID

https://orcid.org/0000-0001-5746-3354

Correspondencia:

Marcelo Bernardin M

Email:doctorbernardin@gmail.com 
senta el caso de un paciente sin comorbilidades que se operó de reparación artroscópica del manguito rotador y presentó daño neurológico postoperatorio. Inicialmente se sospechó que el daño había sido producido durante la técnica anestésica, sin embargo, el estudio adecuado demostró que el daño se relacionaba con la técnica quirúrgica. Conclusión: Existen factores que influyen en la posibilidad de daño neurológico asociado a bloqueos de nervios periféricos que pueden ser propios del paciente, de la técnica anestésica o quirúrgicos, siendo estos últimos los más relevantes. En un paciente con clínica compatible debe realizarse resonancia magnética del tejido neural de alta resolución de forma inmediata; estudio de conducción neural sensitiva y electromiografía a las 4 semanas de evolución por un neurólogo experto.

\section{Caso clínico}

$\mathrm{H}$ ombre, 53 años, tabáquico, sufre caída de 2 metros, fracturándose el troquíter con ruptura de tendón supraespinoso. Manejado con analgésicos e inmovilización por 3 semanas.

Al mes se somete a reparación artroscópica. Bajo sedación, se instala catéter interescalénico ecoguiado, neuroestimulador y baja resistencia a la inyección. Punción fuera de plano, punta de la aguja ubicada entre $\mathrm{C} 5, \mathrm{C} 6$ y escaleno medio. Se administran $10 \mathrm{ml}$ de Chirocaína 0,25\% y lidocaína $1 \%$ y se avanzó catéter $4 \mathrm{~cm}$ más allá de la punta. Luego se administra anestesia general. En protocolo destaca procedimiento laborioso e instalación de 7 portales.

En postoperatorio se indicó PCA Chirocaína 0,1\% 7/5/20. $24 \mathrm{~h}$ postoperado desarrolla omalgia severa, EVA 10/10, irradiado al dorso del hombro y región lateral del brazo y antebrazo, con respuesta parcial a opioides y excelente respuesta a bolos de lidocaína $1 \% 10 \mathrm{ml}$ por catéter.

$48 \mathrm{~h}$ postoperado es dado de alta, EVA 4/10 con bomba elastomérica de Chirocaína 0,12\% a $10 \mathrm{ml} / \mathrm{h}$.

A 10 horas del alta, reingresa con dolor EVA 8/10, urente asociado a hipoestesia. Se administra bolo de $20 \mathrm{ml}$ de Chirocaína al 0,25\% con buena respuesta. A las $72 \mathrm{~h}$ postoperado, tratante solicita retiro del catéter y se indica PCA de morfina, AINES y pregabalina. Mantuvo EVA 5/10.

A la semana, la resonancia no muestra alteraciones y traumatólogo descarta lesión quirúrgica, indicando complicación anestésica: "Plexitis".

En la segunda semana se inicia kinesioterapia y fisioterapia. Equipo de dolor crónico lo manejó con ketamina, pulsos de lidocaína semanal, pregabalina, amitriptilina, lamotrigina y Celebra. Anestesiólogo solicita resonancia de plexo que paciente rechaza por dolor.
A la tercera semana, estudio de conducción nerviosa muestra lesión del nervio axilar, realizándose infiltración corticoidal del nervio, con mejoría parcial.

A los 3 meses con EVA 3/10 y descargas intermitentes e inicia rehabilitación por hombro congelado. Al año persiste con dolor intermitente, neuropático, con recuperación parcial.

\section{Definición de injuria de nervio periférico}

Dolor, parestesias, déficit sensorial, motor u otra anormalidad neurológica, nuevo o empeorado, que persiste más allá del tiempo de acción del anestésico local.

La mayoría son transitorias y autolimitadas. Más de 12 meses se considera permanente.

La incidencia es variable y el $99 \%$ se recupera al año. Las lesiones serias tienen una incidencia de 2,4:10.000 bloqueos.

Variables que determinan frecuencia:

1. Tamaño de estudios.

2. Procedimiento quirúrgico.

3. Técnica de bloqueo y extremidad.

4. Habilidad anestésica.

5. Estado de conciencia.

6. Exclusión (enfermedades previas).

7. Definición (nuevo o empeoramiento del preexistente; injuria anestésica o quirúrgica).

8. Método de evaluación (por anestesiólogo, por paciente, cirujano o voluntario).

9. Tiempo de seguimiento.

Sites[1] publicó un estudio de 12.668 bloqueos ecoguiados. La incidencia de intoxicación por AL fue infrecuente, sin embargo, el daño neurológico a 6 meses fue de 0,9:1.000.

Los bloqueos con mayor incidencia de daño fueron: interescalénico e interescalénico continuo, 
12:12.000. Factores determinantes: volúmenes mayores a $30 \mathrm{ml}$ con concentraciones de chirocaína o bupivacaína de 0,5\% asociado a aditivos y comorbilidades como hipertensión, diabetes, esclerosis múltiple.

Respecto a closed claims[2] de los últimos 20 años, más de la mitad de las lesiones fueron transitorias y sólo un tercio permanentes. El plexo braquial tuvo mayor incidencia de lesiones permanentes.

\section{Anatomía neural}

Las fibras se rodean de endoneuro, varias formarán fascículos rodeados de perineuro. Varios fascículos forman el nervio, que está rodeado de epineuro. Existen además vasos intrínsecos y extrínsecos, que mantienen la homeostasis y perfusión. Frente a fenómenos isquémicos, habrán deterioros funcionales.

Moayeri[3] publicó un estudio histológico del plexo a niveles: interescalénico, supraclavicular, infraclavicular proximal y distal, confirmando que el tejido conectivo aumenta hacia distal, por tanto, a proximal el tejido neural está más compacto, determinando menor latencia para bloqueos, pero mayor susceptibilidad a injurias.

Además, existen macrófagos, fibroblastos y astrocitos, que regulan la conducción y perfusión.

A nivel de las raíces, emergen fascículos de diferentes nervios, combinándose hacia distal, por lo que la respuesta motora a la neuroestimulación resulta variable, no así cuando se estimula un nervio terminal.

\section{Neuropatología}

Mizisin[4], revisó la homeostasis del nervio, existiendo un microambiente autorregulable, que puede alterarse por diferentes injurias: trauma, enfermedad o tóxicos.

El anestésico altera la permeabilidad generando hipotonía, edema e isquemia intrafascicular, principalmente en fibras delgadas (dolor y temperatura) y menos en motoras y propioceptivas, produciendo vasoconstricción indirecta, aumentando el calcio intracelular, alterando el metabolismo y produciendo apoptosis.

\section{Existe mayor toxicidad al mezclar anestésicos locales sobre rangos clínicos y a mayor tiempo de exposición}

El torniquete, afecta a fibras de mayor diámetro (motoras), debiendo utilizarse máximo dos horas. Más de tres, hay daño axonal, y seis lesiones irreversibles.
Mecanismos fisiopatológicos: Campbell[5] describió mecanismos como laceración, estiramiento o atrapamiento, daño vascular asociado a isquemia y hemorragia, presión extra o intraneural, daño químico como neurotoxicidad por anestésicos locales, alcoholización y daño térmico por radiación, quemadura y electricidad

Clasificaciones de daño: neuropraxia, alteración de funcionalidad; axonotmesis, destrucción del axón y neurotmesis, destrucción del nervio.

En la axonotmesis, la injuria atrae macrófagos desmielinizando el axón manteniendo los tubos neurales (degeneración walleriana). Como respuesta en el soma aumenta la secreción de elementos neurotróficos fomentando la regeneración, pudiendo canalizarse a través del tubo neural volviendo a inervar o perdiéndose, generando atrofia y la formación de un neuroma a distal. La regeneración es muy lenta, $1 \mathrm{~mm} /$ día, por tanto, una lesión proximal tardará en recuperarse.

La degeneración walleriana producirá alteración de permeabilidad, aumentando la presión intrafascícular, con isquemia y disfunción, contribuyendo al daño.

En neurotmesis con sección completa no habrá reinervación y podría repararse con anastomosis quirúrgica término terminal o injerto de nervio.

\section{Factores de riesgo}

I. Paciente: masculino, tabaquismo, hipertensión, edad avanzada (microangiopatía), enfermedades neurológicas preexistentes (esclerosis múltiples, radiculopatías compresivas, estenorraquis, diabéticos y neuropatía inflamatoria postquirúrgica).

Enfermedad neurológica preexistente: teoría "doble crush", donde aparece una nueva noxa sobre daño preexistente. Presentada sobre enfermedades subclínicas como diabetes.

Las neuropatías inflamatorias postquirúrgicas: Ahn[6] reporta un caso de artroplastía bilateral de cadera con anestesia mixta. En el postoperatorio inmediato desarrolló un déficit neurológico bilateral que desapareció posteriormente al uso de corticoides. Este se produce por respuesta inmunológica idiopática al estrés quirúrgico, bloqueos regionales, infecciones, síndrome paraneoplásico o trastornos nutricionales. Manifestándose hasta 30 días posteriores, requiere derivación y tratamiento corticoidal precoz.

II. Quirúrgicos: (88-90\%). Traumas quirúrgicos o estiramientos de plexo, isquemia por torniquete, 
compromiso vascular, inflamación perioperatoria, hematoma intra o extra neural, yesos compresivos o irritación, infecciones postoperatorias, posición intraoperatoria, cirugía cerca del área bloqueada.

Rashld[7] revisó daño neural asociado a cirugía de hombro describiendo un indencia de 1-2\% en manguito rotador, $1-8 \%$ en inestabilidad anterior y $1-4 \%$ en artroplastías.

Las áreas quirúrgicas se relacionan con los nervios axilar, supraescapular, músculocutáneo y subescapular. En artroscopía se utilizan 4 portales, más aumentan el riesgo de daño, así como suturas, tracción excesiva y posiciones extremas mantenidas.

En luxaciones de hombro, la ruptura de la cápsula y ligamentos pueden distender el nervio axilar, generando denervación del deltoides. No es recomendable la realización de bloqueos en estos casos por riesgo de producir un "second crush" sobre este nervio que está regenerándose, y empeorar el pronóstico.

Swiggum[8] publicó sobre la injuria de nervio post artroplastía de hombro con anestesia regional, evaluando si el bloqueo interescalénico se relacionaba con un mayor riesgo. En 1.569 pacientes, 35 presentaron algún déficit (2,2\%), OR de 0,42\% determinando que no hubo aumento de incidencia, y en los casos de injuria, la mayoría se recuperó al año.

En posición de silla de playa, hay riesgo de compresión del cubital y peroneo común. Adicionalmente de sobredistensión del plexo por flexoextensión lateral y anterior de la cabeza. En decúbito lateral también hay riesgo de sobredistensión.

III. Anestésicos: trauma mecánico por agujas y catéter, inyecciones intrafasciculares, injuria isquémica por vasoconstrictores o anestésicos locales en altas concentraciones, edema perineural, toxicidad del anestésico.

Albrecht[9] evaluó la distancia máxima entre punta de la aguja y el nervio en bloqueo interescalénico manteniendo efectividad. Con $20 \mathrm{ml}$ de bupivacaína 0,5\% en escaleno medio, describió un 95\% de efectividad a 1,6 $\mathrm{mm}$ del borde y $50 \%$ a $8 \mathrm{~mm}$, destacando la difusión bajo la fascia prevertebral.

Myers[10] describió la neurotoxicidad de los anestésicos locales, los que alterarían la permeabilidad perineural, provocando vasoconstricción, edema endoneural y subperineural.

Yang[11] analizó la toxicidad del anestésico local sobre las células de Schwann, encontrando una relación directa con concentración y tiempo.

\section{"El daño siempre es multifactorial"}

Evaluación diagnóstica y manejo: (Figura 1).

1. Documentar déficit previo.

2. Identificar disfunción ¿motora o sensitiva?

Figura 1.

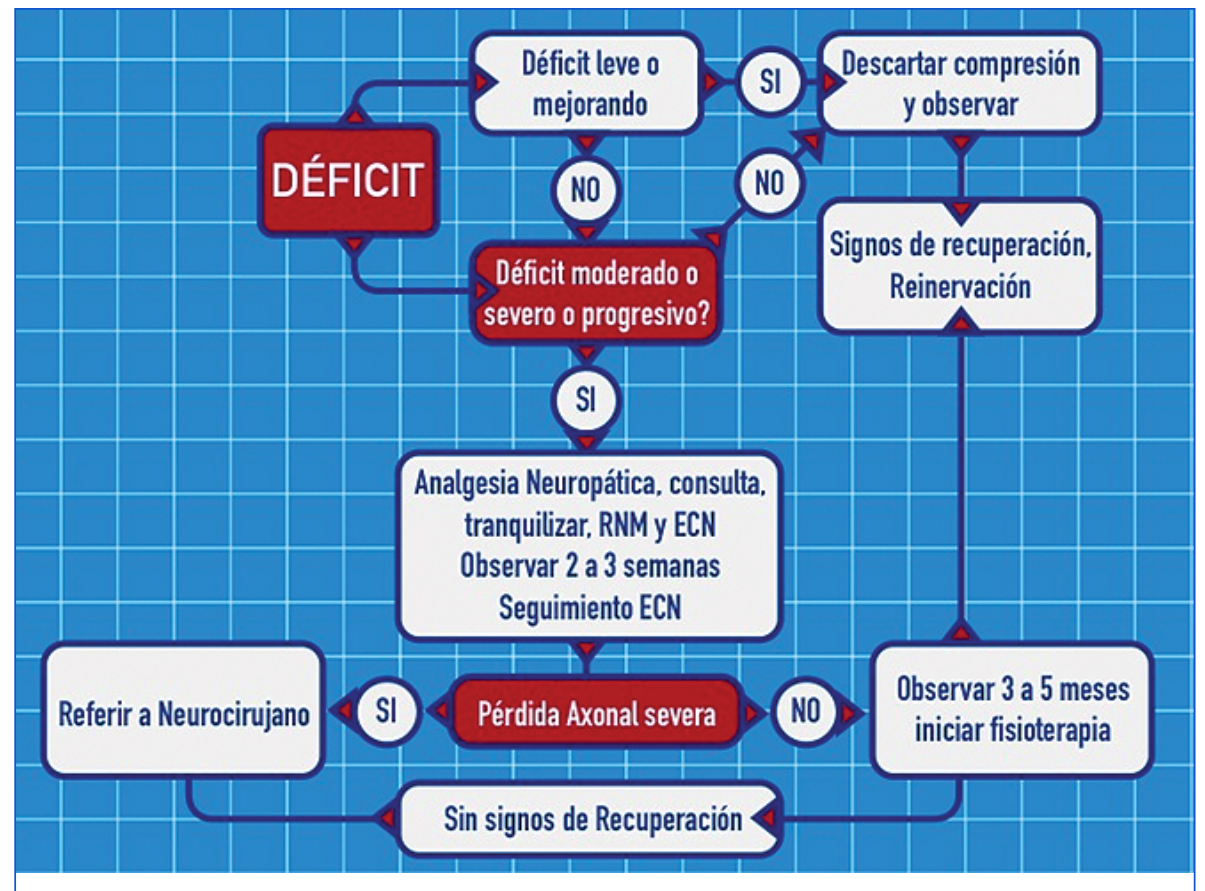


3. Historia: comienzo, severidad, cualidad de síntomas.

4. Evolución.

5. Factores paciente (diabetes, esclerosis múltiple, vasculopatía, quimioterapia).

6. Eventos quirúrgicos: trauma, tracción, sangrado, dificultad en exposición quirúrgica.

7. Examen físico: equímosis en sitio de punción e isquemia distal, coagulopatías, sospecha de infección.

8. Evaluación precoz por neurólogo o neurocirujano para determinar severidad y pronóstico.

9. Exámenes: Resonancia nuclear magnética y estudio electrodiagnóstico (Figura 2).

10. Participar en el seguimiento y monitorizar la evolución.
11. Referir a neurocirujano en caso de lesión completa, disfunción severa sin mejoría clínica, sin evidencia de reinervación a los 4 meses postinjuria (no habrá beneficio después de 12 a 18 meses).

En el caso clínico, la electromiografía y conducción nerviosa mostraron un daño compatible con un compromiso neurogénico parcial en territorio axilar izquierdo, con ocasionales signos de degeneración axonal activos.

\section{Conclusiones}

Se pudo demostrar que el daño fue ocasionado por la técnica quirúrgica.

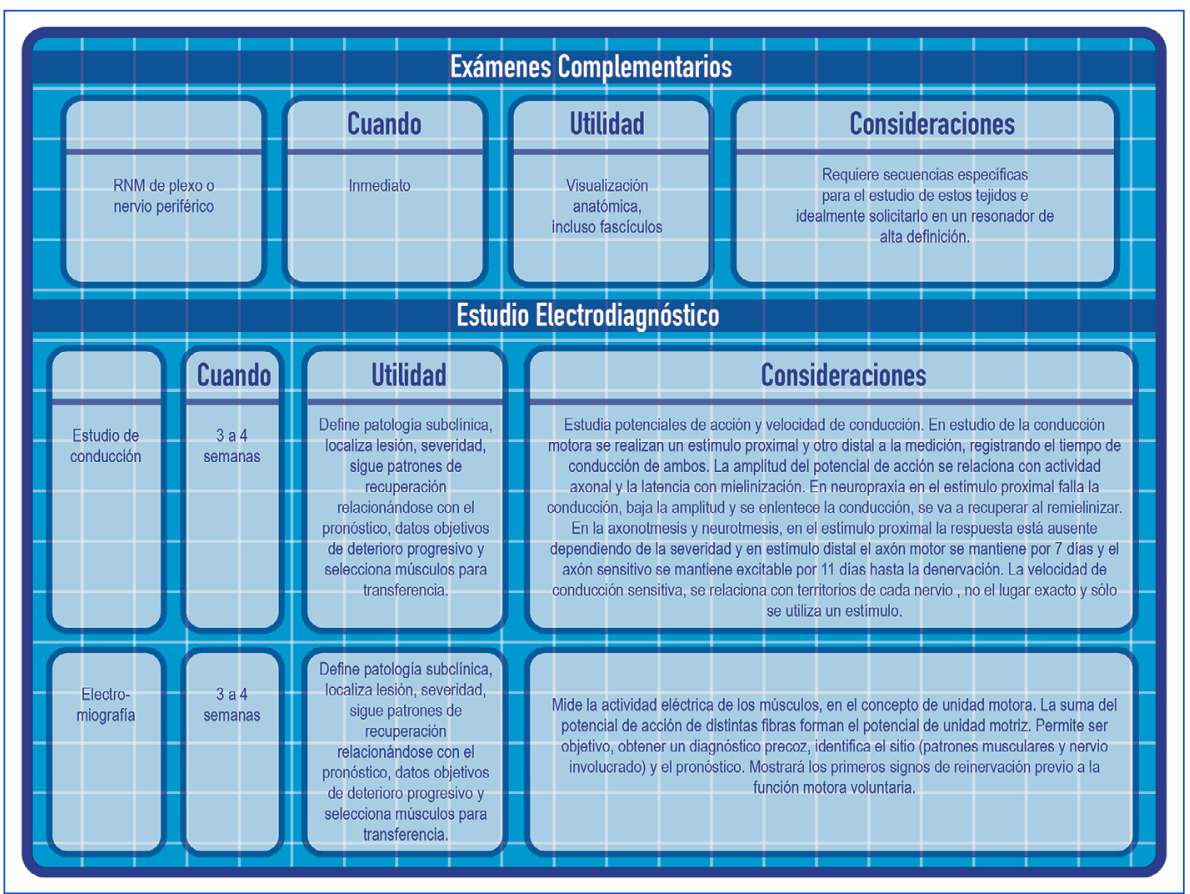

Figura 2.

\section{Referencias}

1. Sites $B D$, Taenzer $A H$, Herrick MD, Gilloon C, Antonakakis J, Richins J, et al. Herrick MD y cols. Incidence of Local Anesthetic Systemis Tpxicity and Postoperative Neurologic Symptoms Associated With
12,668 Ultrasound-Guided Nerve Blocks. Reg Anesth Pain Med. 2012;37(5):478-82. https://doi.org/10.1097/ AAP.0b013e31825cb3d6 PMID:22705953

2. Lee LA, Posner KL, Cheney FW, Caplan RA, Domino KB. Complications associated with eye blocks and peripheral nerve blocks: an american society of anesthesiologists closed claims analysis. Reg Anesth Pain Med. 2008 SepOct;33(5):416-22. https:// doi.org/10.1097/00115550200809000-00004

PMID:18774510 
3. Moayeri N, Bigeleisen PE, Groen GJ. Quantitative architecture of the brachial plexus and surrounding compartments, and their possible significance for plexus blocks. Anesthesiology. 2008 Feb;108(2):299-304. https://doi.org/10.1097/01. anes.0000299433.25179.70 PMID:18212575

4. Mizisin AP, Weerasuriya A. Homeostatic regulation of the endoneurial microenvironment during development, aging and in response to trauma, disease and toxic insult. Acta Neuropathol. 2011 Mar;121(3):291-312. https:// doi.org/10.1007/s00401-0100783-x PMID:21136068

5. Campbell WW. Evaluation and management of peripheral nerve injury. Clin Neurophysiol. 2008 Sep;119(9):1951-65. https://doi.org/10.1016/j. clinph.2008.03.018 PMID:18482862
6. Ahn KS, Kopp SL, Watson JC, Scott KP, Trousdale RT, Hebl JR. Postsurgical inflammatory neuropathy. Reg Anesth Pain Med. 2011 Jul-Aug;36(4):403-5. https://doi.org/10.1097/

AAP.0b013e31821e6503 PMID:21654554

7. Rashld A, Abdul-Jabar H, Lam F. Nerve injury associated with shoulder surgery. Curr Orthop. 2008;22(4):284-8. https://doi.org/10.1016/j. cuor.2008.04.009.

8. Sviggum HP, Jacob AK, MantiIla CB, Schroeder DR, Sperling JW, Hebl JR. Perioperative nerve injury after total shoulder arthroplasty: assessment of risk after regional anesthesia. Reg Anesth Pain Med. 2012 Sep-Oct;37(5):490-4. https://doi.org/10.1097/ AAP.0b013e31825c258b PMID:22705952

9. Albrecht E, Kirkham K, Taffé $P$, Endersby RV, Chan VW, Tse C, et al. Taffé P y cols. The Maximum Effective Needle-to Nerve Distance for Ultrasound-Guided Interescalene Block. Reg Anesth Pain Med. 2014;39(1):56-60. https://doi.org/10.1097/ AAP.0000000000000034 PMID:24310046

10. Myers RR, Kalichman MW, Reisner LS, Powell HC. Neurotoxicity of local anesthetics: altered perineurial permeability, edema, and nerve fiber injury. Anesthesiology. 1986 Jan;64(1):29-35. https:// doi.org/10.1097/00000542198601000-00006 PMID:3942334

11. Yang $S$, Abrahams MS, Hurn PD, Grafe MR, Kirsch JR. Local anesthetic Schwann cell toxicity is time and concentration dependent. Reg Anesth Pain Med. 2011 Sep-Oct;36(5):444-51. https://doi.org/10.1097/ AAP.0b013e318228c835 PMID:21857272 